\title{
Función Suprarrenal en Pacientes con Síndrome Bronquial Obstructivo Tratados con Corticoides Sistémicos (Informe Preliminar)
}

\author{
FRANCISCA ARANCIBIA H. ${ }^{1}$, LINUS HOLMGREEN P. ${ }^{2}$, ALEJANDRO MARTÍNEZ A. ${ }^{3}$ \\ 1. Residente Pediatría. Pontificia Universidad Católica de Chile. \\ 2. Departamento de Pediatría, Unidad de Respirología. Pontificia Universidad Católica de Chile. \\ 3. Departamento de Pediatría, Unidad Endocrinología, Pontificia Universidad Católica de Chile.
}

\begin{abstract}
Adrenal Function in Patients with Acute Bronchial Obstruction Treated with Systemic Corticosteroids: A Preliminary Report
\end{abstract}

Introduction: Systemic corticosteroids are widely used in patients with acute bronchial obstruction (ABO). It has been recommended that such treatment last a maximum of 5 days to avoid adverse effects. Suppression of the adrenal axis under these conditions among children has not been evaluated. Objective: Assessment of the hypothalamic pituitary adrenal axis (HHS) function after use of systemic steriods in children after a 5-7 day use of corticoids, utilizing the micro ACTH test. Method: Prospective observational study conducted in the Department of Pediatrics, Hospital Clínico Universidad Católica de Chile. ACTH test used with microdosis $\left(1 \mathrm{mcg} / 1.73 \mathrm{~m}^{2}\right)$. A normal response was determined if cortisol post-ACTH $>20$ mcg/dL. Results: 7 patients were recruited, median age was 4 years 4 months (range 4 months to 8 years). The test was perfomed within an average of 72 hours after discontinuation of prednisone. All patients had normal basal cortisol values, with an average value of $6.5 \mathrm{mcg} / \mathrm{dl}$, range 1 to $9 \mathrm{mcg} / \mathrm{dl}$. The ACTH test yielded a response of cortisol levels with an average value of $13.6 \mathrm{mcg} / \mathrm{dL}$ and a range of 3.7 to $20 \mathrm{mcg} / \mathrm{dL}$. Five of the 7 patients presented an abnormal response. Conclusions: Our results suggest that the adrenal response may be reduced in children who have been treated with systemic steroids for $A B O$ even if managed during short periods of time.

(Key words: Adrenal function, acute bronchial obstruction, corticosteroids).

Rev Chil Pediatr 2009; 80 (4): 347-353

\section{RESUMEN}

Introducción: Los corticoides sistémicos son ampliamente utilizados en pacientes con crisis bronquiales obstructivo (CBO). Se ha recomendado un máximo de 5 días para evitar efectos adversos. No ha sido evaluado si tratamientos por cursos cortos pueden suprimir el eje adrenal. Objetivo: Evaluar la función del

Trabajo recibido el 02 de marzo de 2009, aceptado para publicación el 08 de junio de 2009.

Correspondencia a:

Dra. Francisca Arancibia H.

E-mail: farancib@gmail.com 
eje hipotálamo hipófisis suprarrenal (HHS) mediante la prueba de microdosis de ACTH en pacientes con CBO que recibieron corticoides sistémicos entre 5 a 7 días. Diseño: Estudio observacional prospectivo realizado en el Servicio de Pediatría del Hospital Clínico de la Universidad Católica de Chile. Prueba de ACTH con microdosis $\left(1 \mathrm{mcg} / 1,73 \mathrm{~m}^{2}\right)$. Se consideró como respuesta normal una respuesta de cortisol postestímulo $>20 \mathrm{mcg} / \mathrm{dL}$. Resultados: Fueron reclutados 7 pacientes, mediana de edad de 4 años 4 meses (rango 4 meses a 8 años). Los resultados fueron obtenidos con una mediana de 72 horas de suspendidos los corticoides (prednisona). Todos los pacientes presentaban valores de cortisol basal normal promedio de $6,5 \mathrm{mcg} / \mathrm{dl}$ (rango 1 a $9 \mathrm{mcg} / \mathrm{dl})$. La respuesta de cortisol postestímulo fue de 13,6 mcg/dL (3,7 a $20 \mathrm{mcg} /$ dL), cinco de los 7 pacientes presentaron respuesta anormal. Conclusiones: La respuesta adrenal puede estar disminuida en pacientes que usan corticoides sistémicos por cursos cortos por CBO.

(Palabras clave: Función adrenal, síndrome bronquial obstructivo, corticoides).

Rev Chil Pediatr 2009; 80 (4): 347-353

\section{Introducción}

Los glucocorticoides son un grupo de agentes antiinflamatorios extremadamente potentes usados en forma sistémica e inhalatoria en el tratamiento del asma y del síndrome bronquial obstructivo $^{1-2}$. Ellos bloquean la respuesta pulmonar tardía y el desarrollo de hiperreactividad bronquial ${ }^{3}$.

A pesar de ciertas controversias sobre su eficacia, rutas de administración y dosis, la evidencia sugiere que ${ }^{4,5}$ : requieren por lo menos entre 6 a 24 horas para actuar; las vías intravenosa $u$ oral parecen ser equivalentes; un plan de 7 a 10 días de administración a partir del alta de la urgencia/emergencia reduce significativamente las recaídas. El Ministerio de Salud de Chile ${ }^{6}$ recomienda el uso de prednisona oral 1-2 $\mathrm{mg} / \mathrm{kg}$ en dosis única o corticoide parenteral (hidrocortisona, metilprednisolona o betametasona) y que los pacientes enviados a su casa al cabo de la segunda hora de tratamiento deben recibir prednisona $1-2 \mathrm{mg} / \mathrm{kg} / \mathrm{día}$, dividida cada 12 horas o en dosis única matinal, hasta el control al día siguiente con un máximo de uso de prednisona de 5 días.

La hipótesis de nuestro trabajo es que en individuos con crisis bronquiales obstructivas, el uso de corticoides sistémicos por 5 a 7 días se asocia a una respuesta alterada a la prueba de ACTH con microdosis realizada después de las 48 a 72 horas de haber suspendido los esteroides.

\section{Pacientes y Método}

\section{Diseño}

Estudio observacional prospectivo.

\section{Lugar}

Servicio de Pediatría de la Pontificia Universidad Católica de Chile, hospitalizado en sala o visto en forma ambulatoria en CEDIUC o Urgencia UC.

\section{Pacientes}

Los pacientes incluidos en el estudio debieron cumplir con los siguientes criterios de inclusión: a) Paciente en edad pediátrica con SBO que hayan requerido uso de corticoides endovenoso $\mathrm{y} / \mathrm{u}$ oral durante mayor o igual a 5 días hasta 7 días; b) Eutrófico. Criterios de exclusión: a) Antecedente de uso de corticoides (inhalado, oral o tópico) por lo menos un mes antes de la evaluación; b) Utilizar medicamentos que interfieran con la esteroidogénesis $(\mathrm{Ej}$. Ketoconazol); c) Pacientes con lesión de línea media del SNC y/o deficiencia conocida de ACTH.

\section{Protocolo del Estudio}

Al cumplir 5-6 ó 7 días con tratamiento con glucocorticoides los pacientes fueron evaluados por residente de pediatría y/o endocrinólogo pediátrico del Departamento de Pediatría de la Pontificia Universidad Católica de Chile. Se 
registró el tipo, dosis y número de días de tratamiento con corticoides.

Entre 48 a 72 horas de suspendido el tratamiento con corticoide se realizó medición de cortisol basal y de hormona adreno corticotrófica $(\mathrm{ACTH})$ y 30 minutos más tarde se realizó test de microdosis de ACTH.

\section{Prueba de ACTH con Microdosis}

Se citó a los pacientes con un ayuno nocturno de mínimo de 4 horas. Entre las 8-9:00 am se instalaba una cánula endovenosa previa aplicación de crema anestésica en zona a puncionar, luego de un reposo de diez minutos se tomaba una muestra de sangre basal para determinar ACTH y cortisol. La Prueba de ACTH de microdosis se realizó determinando los niveles de cortisol plasmático 30 minutos después de la inyección endovenosa de cosintropina (ACTH sintética (1-24), Cortrosyn ${ }^{\circledR}$, Basel, Suecia, en dosis de $1,0 \mathrm{mcg}$ ó $0,5 \mathrm{mcg}$ por $\left.1,73 \mathrm{~m}^{2}\right)^{7,8}$.

\section{Ensayo para Determinar Muestra de ACTH y Cortisol}

Tipo de examen inmunoensayo automatizado con detección por quimioluminiscencia (Immulite 2000 DCP).

\section{Definición de Insuficiencia Suprarrenal}

Se consideró como insuficiencia suprarrenal niveles de cortisol sérico $<20 \mathrm{~g} / \mathrm{dL}$ a los 30 minutos post $1 \mu \mathrm{g} / 1,73 \mathrm{~m}^{2} 9,10$. A los pacientes con respuesta anormal se les realizó un segundo examen entre los 10 y 15 días de suspendidos los corticoides para evaluar recuperación del eje.

\section{Seguimiento de los Pacientes con Respuesta Anormal}

A los pacientes con respuesta anormal se les indico un segundo test de microdosis de ACTH entre el día 10 y 15 de suspendidos los corticoides para evaluar recuperación del eje.

Además fueron seguidos en forma telefónica por residente de pediatría para determinar la presencia de cuadros intercurrentes que los pusieran en situación de stress y para determinar la presencia de síntomas de insuficiencia suprarrenal. De ser así se les citaba para evaluación médica, se les indicaba cortisol en dosis de stress $\left(40 \mathrm{mg} / \mathrm{m}^{2} /\right.$ día $)$ y se les realizaba seguimiento.

\section{Comité de ética}

Este trabajo fue aprobado por el Comité de Ética de la Pontificia Universidad Católica de Chile. Los padres debían firmar previa aceptación del estudio un consentimiento informado. A los niños mayores de siete años se les solicitó asentimiento informado.

\section{Financiamiento}

El estudio cuenta con financiamiento de SOCHIPE (proyecto $N^{\circ}$ P2008007) y del Centro Investigaciones Médicas PUC (Proyecto Becado PG30/08).

\section{Estadística}

El análisis estadístico fue realizado con el programa computacional SPSS versión 10.0 (SPSS, Inc., Chicago, IL). Los resultados fueron expresados en mediana (rango) y en porcentaje de sujetos con respuesta anormal. Se realizo test para variables pareadas para evaluar diferencias de cortisol del primer test y el de control.

\section{Resultados}

Hasta el momento, se han reclutado siete pacientes, con una mediana de edad de 4 años 4 meses (rango; 4 meses a 8 años). Cuatro pacientes de sexo masculino. Sólo 2 pacientes requirieron hospitalización durante la crisis bronquial obstructiva, ninguno con crisis bronquial grave que hubiera requerido manejo en unidad de paciente critico (tabla 1).

Todos usaron como tratamiento corticoidal prednisona con una dosis promedio de $1,6 \mathrm{mg} /$ $\mathrm{kg} /$ día (rango; 1,2 a $2 \mathrm{mg} / \mathrm{kg} /$ día), equivalente a $197 \mathrm{mg} / \mathrm{m}^{2} /$ día de cortisol (10 a 20 veces la dosis fisiológica), durante 5 a 7 día con una mediana de 5 días (tabla 2).

Los resultados fueron obtenidos con una mediana de 72 horas de suspendidos los corticoides (rango; 48 a 96 horas). En la muestra basal la ACTH promedio fue $15 \mathrm{pg} / \mathrm{mL}$ (rango; 5 a $53 \mathrm{pg} / \mathrm{mL}$ ), el cortisol basal en $100 \%$ de los pacientes fue normal con un valor 
Tabla 1. Descripción de características de pacientes, corticoides utilizados y resultados de test medidos

\begin{tabular}{|c|c|c|c|c|c|c|c|c|c|c|c|c|c|c|}
\hline 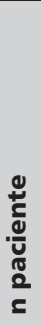 & 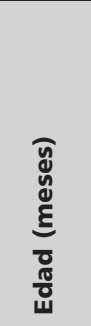 & 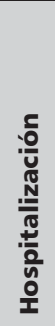 & 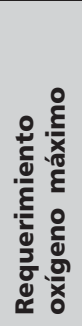 & 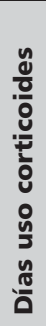 & 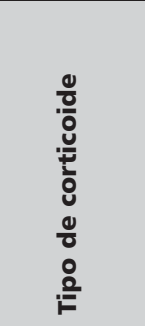 & 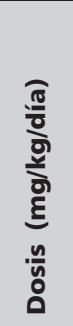 & 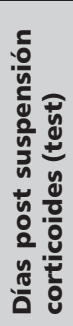 & 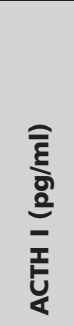 & 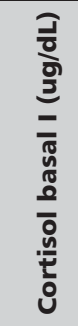 & 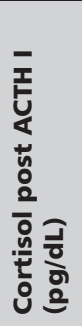 & 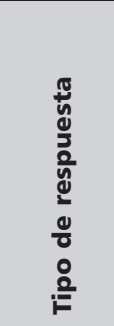 & 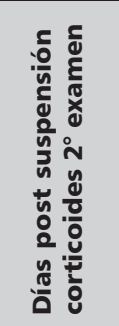 & 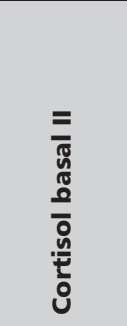 & 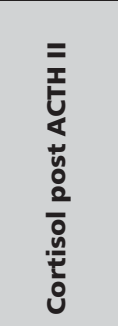 \\
\hline 1 & 8,20 & Si & $1 \mathrm{Lt}$ & 5 & Prednisona & 1,36 & 3 & 14 & 5,5 & 13,6 & Anormal & 10 & 4,3 & 15,7 \\
\hline 2 & 96,57 & No & $21 \%$ & 5 & Prednisona & 1,41 & 3 & $<5$ & 3,9 & 14,8 & Anormal & no quizo & no quizo & no quizo \\
\hline 3 & 4,13 & No & $21 \%$ & 6 & Prednisona & 1,8 & 2 & 50,4 & 6,7 & 15,8 & Anormal & 12 & 4,7 & 24,4 \\
\hline 4 & 52,53 & Si & $3 \mid \mathrm{t}$ & 7 & prednisona & 1,8 & 2 & 6,9 & $<1$ & 3,7 & Anormal & 12 & 9.8 & 18 \\
\hline 5 & 65,30 & No & $21 \%$ & 5 & Prednisona & 1,6 & 3 & 52,2 & 6,5 & 20 & Normal & ---- & 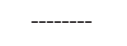 & ------ \\
\hline 6 & 8,70 & No & $21 \%$ & 5 & Prednisona & 2 & 3 & 53,2 & 7 & 8,9 & Anormal & 13 & 3,3 & 15,5 \\
\hline 7 & 90,70 & no & $21 \%$ & 5 & Prednisona & 1,3 & 4 & 16,1 & 8,8 & 12,9 & Anormal & 10 & 9,5 & 20,5 \\
\hline
\end{tabular}

Tabla 2. Dosis y tiempo de duración de la prednisona

\begin{tabular}{lll}
\hline & Mediana & Rango \\
\hline Tiempo de uso & 5 días & 5 a 7 días \\
Dosis promedio (Prednisona) & $1,6 \mathrm{mg} / \mathrm{kg} /$ día & 1,2 a $2 \mathrm{mg} / \mathrm{kg} /$ día \\
Equivalente de cortisol & $197 \mathrm{mg} / \mathrm{m}^{2} /$ día & 136 a $220 \mathrm{mg} / \mathrm{m}^{2} /$ día* \\
\hline
\end{tabular}

*13 a 20 veces la dosis fisiológica (=10 mg/m²/día).

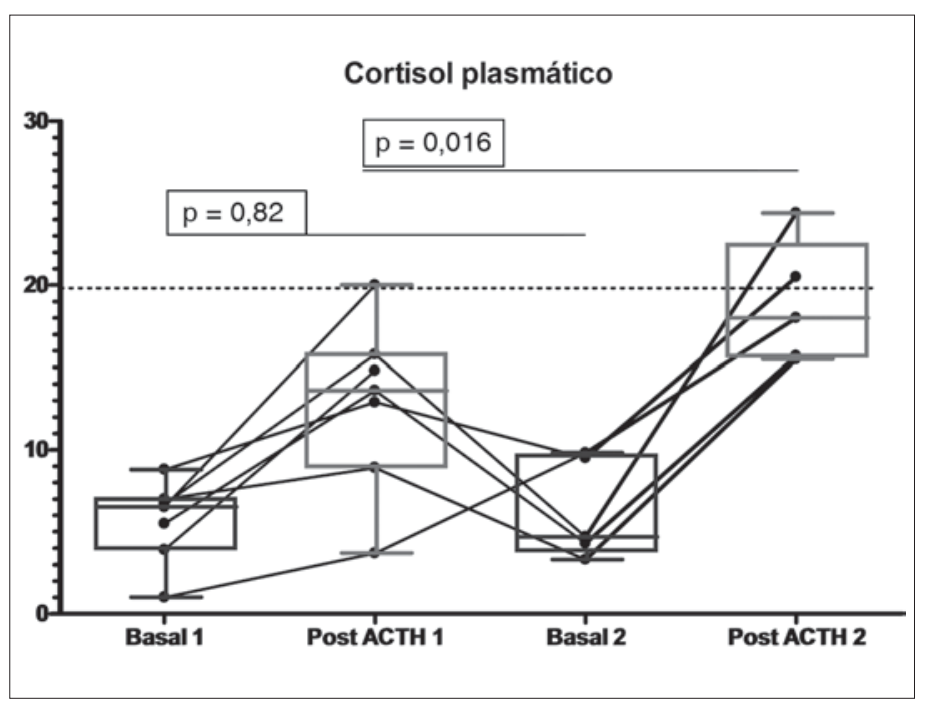

Figura 1. Cortisol basal y post test microdosis ACTH. mediana de 6,5 mcg/dL (rango; 1 a $9 \mathrm{mcg} / \mathrm{dL}$ ) y el cortisol postestímulo fue 13,6 mcg/dL (rango; 3,7 a $20 \mathrm{mcg} / \mathrm{dL}$ ). Una respuesta anormal, cortisol post estímulo $<20 \mathrm{mcg} /$ dL, se observó en seis de los siete pacientes estudiados (figura 1).

Durante el seguimiento telefónico de los pacientes con respuesta anormal sólo 1 de los 7 presentó un cuadro intercurrente en el período observado (Síndrome diarreico agudo con deshidratación moderada). Este paciente recibió tratamiento con cortisol en dosis de stress $\left(50 \mathrm{mg} / \mathrm{m}^{2} /\right.$ día) durante los tres días de duración de su diarrea. El resto de los pacientes se mantuvo asintomático por lo que no requirió suplementación.

A cinco de los 6 pacientes con respuesta anormal se les realizó un segundo examen, uno no acepto repetirlo. El examen fue repetido a una mediana de 12 días de suspendidos los corticoides (rango 10 a 13 días). Todos los pacientes presentaron niveles de cortisol basal normal con una mediana de 
4,7 $\mu \mathrm{g} / \mathrm{dL}$ (rango 3,3 a 9,8 $\mu \mathrm{g} / \mathrm{dL}$ ), no hubo una diferencia estadísticamente significativa con respecto a los niveles de cortisol basal del primer examen (figura 1).

Los niveles de cortisol post ACTH fueron normales en 2 de los 5 pacientes, pero es importante destacar que si mostraron un aumento estadísticamente significativo con respecto al primer examen, el valor mediana de ACTH post estímulo fue de $18 \mu \mathrm{g} / \mathrm{dL}$ (Rango 15,5 a $24,4 \mu \mathrm{g} / \mathrm{dL}$ ). Estos pacientes fueron seguidos telefónicamente y todos evolucionaron clínicamente estables, ninguno con síntomas que hicieran sospechar insuficiencia suprarrenal.

\section{Discusión}

En este informe preliminar presentamos los resultados de la evaluación del eje hipotálamo hipofisiario suprarrenal en un grupo seleccionado de pacientes que usaron corticoides sistémico en dosis supra-fisiológica por un período de 5 a 7 días. La mayoría de ellos mostró una respuesta insuficiente a la Prueba de ACTH con microdosis incluso tras tres días de haber suspendido el tratamiento esteroidal. La importancia clínica de este fenómeno no fue relevante en relación a síntomas o signos de insuficiencia suprarrenal reportados por los pacientes. Sin embargo, un sujetó presentó deshidratación moderada secundaria a una diarrea aguda en la primera semana después de haber suspendido la prednisona, considerando que este paciente tenía una respuesta a ACTH insuficiente, se indicó cortisol en dosis de estrés.

Dentro de los métodos utilizados para detectar supresión de la función suprarrenal se ha utilizado la medición de la concentración de cortisol plasmático matinal ${ }^{11}$. Este método se ha usado en pacientes pediátricos asmáticos usuarios de corticoides inhalados; y se ha observado que esta evaluación tiene una gran sobreposición de resultados con respecto a pacientes controles no usuarios de corticoides, por este motivo no se recomienda su uso para discriminar insuficiencia suprarrenal ${ }^{12}$.

La determinación sérica de cortisol durante 12 ó 24 horas podría ser más apropiada para evaluar la supresión suprarrenal. Sin embargo, la determinación del área bajo la curva por medio de mediciones horarias de los niveles de cortisol durante el día y la noche requiere de hospitalización, con costos económicos y emocionales. La determinación de cortisol urinario libre no es adecuada para la detección de insuficiencia suprarrenal ${ }^{18}$. La evaluación del eje hipotálamo hipófisis suprarrenal requiere de pruebas funcionales como la inducción de hipoglicemia usando insulina en la "Prueba de Tolerancia a la Insulina" (PTI), ésta se considera como la "Prueba de Oro", pero los riesgos de su uso lo restringen a centros especializados. La "Prueba con Metirapone" ha sido recomendada en lugar del PTI y ha mostrado una baja tasa de efectos adversos. Es más sensible que la Prueba con dosis estándar de ACTH (250 mcg) en detectar grados menores de insuficiencia suprarrenal secundaria a corticoides exógenos, pero su experiencia es limitada en niños asmáticos. La Prueba con dosis estándar de ACTH (250 mcg) carece de especificidad y puede dar una falsa respuesta normal en casos de supresión leve del eje HHA, la que si hacen evidentes con la $\mathrm{PTI}^{13}$. La prueba con microdosis de ACTH puede detectar deficiencias de ACTH con cierta precisión ${ }^{14,15}$. Esta prueba es de menor costo, segura y simple de realizar. $\mathrm{La}$ Prueba con microdosis de ACTH tiene sensibilidad intermedia entre las pruebas dinámicas (Prueba con Metirapone, PTI y de dosis estándar de $\mathrm{ACTH}$ ) y es capaz de detectar deficiencias en la secreción de cortisol que no son detectadas por la prueba de dosis estándar ${ }^{16}$.

Se describe que al usar corticoides sistémicos en cursos cortos, entre cinco hasta siete días, no es necesario reducir progresivamente la dosis para evitar la insuficiencia suprarrenal, pero existen reportes clínicos que muestran que pacientes con función adrenal disminuida ${ }^{17-19}$ pueden llegar a presentar crisis de insuficiencia suprarrenal grave ${ }^{20-22}$. Es importante detectar supresión del eje hipofisario-suprarrenal para poder prevenir eventuales efectos adversos como convulsiones, hipoglicemia, coma e incluso muerte; especialmente si hay un estrés intercurrente $^{23-25}$.

Si bien en este reporte existe un número reducido de pacientes evaluados, los resultados muestran que el uso de corticoides sistémicos 
de cursos cortos se asocia a una respuesta inadecuada de la glándula suprarrenal a la Prueba con microdosis de ACTH. En este grupo no se reportó repercusión clínica aparente de este fenómeno. Sin embargo, sí podría ser relevante en caso de un evento intercurrente inmediato a la suspensión de corticoides, pudiendo el paciente requerir cortisol en dosis de estrés durante un corto tiempo.

Creemos que este trabajo no sólo es importante en pacientes con CBO sino también en otros sujetos con enfermedades que son tratados con corticoides de potencia y dosis mayor a la fisiológica como patología oncológica e inmune en quienes se debería tener un alto índice de sospecha de insuficiencia suprarrenal, especialmente considerando que los síntomas y signos de ésta son poco específicos, pero su tratamiento en forma oportuna puede no sólo permitir una mejor adaptación al estrés sino incluso prevenir la muerte.

\section{Referencias}

1.- Barnes PJ: Inhaled glucocorticoids for asthma. N Engl J Med 1995; 332: 868.

2.- Barnes PJ, Adcock IM: How do corticosteroids work in asthma. Ann Intern Med 2003; 139: 359.

3.- Cockcroft DW, Murdock KY: Comparative effects of inhaled salbutamol, sodium cromoglycate, and beclomethasone dipropionate on allergen-induced early asthmatic responses, late asthmatic responses, and increased bronchial responsiveness to histamine. J Allergy Clin Immunol 1987; 79: 734.

4.- Rodrigo G, Rodrigo C: Corticosteroids in the emergency department therapy of acute adult asthma. An evidence based evaluation. Chest 1999; 116: 28595.

5.- Rowe BH, Spooner C, Ducharme FM, Bretzlaff JA, Bota $G W$ : Early emergency department treatment of acute asthma with systemic corticosteroids (Cochrane review). In: Lasserson T, Picot J. The Cochrane library. Oxford: Update Software; 2001.

6.- Ministerio de Salud: Guía Clínica Infección Respiratoria Aguda Baja de Manejo Ambulatorio en menores de 5 años. 1st Ed. Santiago: Minsal, 2005.

7.- Dickstein G, Shechner C, Nicholson WE, et al: Adrenocorticotropin stimulation test: Effects of basal cortisol level, time of day, and suggested new sensitive low dose test. J Clin Endocrinol Metab 1991; 72: 773.

8.- Crowley S, Hindmarsh PC, Holownia P, et al: The use of low doses of ACTH in the investigation of adrenal function in man. J Endocrinol 1991; 130: 475.

9.- Thaler LM, Blevins LS Jr: The low dose (1-microg) adrenocorticotropin stimulation test in the evaluation of patients with suspected central adrenal insufficiency. J Clin Endocrinol Metab 1998; 83 (8): 2726-9. Review.

10.- Sarthi M, Lodha R, Vivekanandhan S, Arora NK: Adrenal status in children with septic shock using lowdose stimulation test. Pediatr Crit Care Med 2007; 8 (1): 23-8.

11.- Eid N, Morton R, Olds B, Clark E, Sheilçh S, Eooney $S$ : Decreased morning serum cortisol lcvc!s in children with asthma treated with inhaled fluticasone propionate. Pediatrics 2002; 09: 217-21.

12.- Raux Demay MC, Magny JP, Idres N, Grimfeld A, Le Bouc Y: Use of the low-dose corticotropin stimulation test for the monitoring of children with asthma treated with inhaled corticosteroids.Horm Res 2006; 66 (2): 51-60.

13.- Mayenknecht J, Dieder'ch S, Bahr Plock Inge U, Oelkers $W$ : Comparison of $1 \mathrm{mcg}$ and high dose corticotropin stimulation tests in patients with pituitary ciscase. J Clin Endocrinol Metab 1998; 83: 1558 62.

14.- Dickstein G, Shechner C, Nicholson WE, et al: Adrenocorticotropin stimulation test: effects of basal cortisol level, time of day, and suggested new sensitive low dose test. J Clin Endocrinol Metab 1991; 72: 773 8.

15.- Tordjman, Jaife A, Trostanetskv Y, Greenma Y, Limor $R$, Stern $N$ : Low-dose $(1 \mu \mathrm{g})$ adrenocorticotrophin (ACTH) stimulation as a screening test for impaired hypothalamo-pituitary-adrenal axis function: sensitivity, specificity and accuracy in comparison with the high-dose $(250 \mu \mathrm{g})$ test. Clin Endocrinol 2000; 52 (5): 633-40.

16.- Tordjman K, Jaffe A, Grazas N, et al: The role of the low dose (1 microgram) adrenocorticotropin test in the evaluation of patients with pituitary diseases. J Clin Endocrinol Metab 1995; 80: 1301.

17.- Sizonenko PC: Effects of inhaled or nasal glucocorticosteroids on adrenal function and growth. J Pediatr Endocrinol Metab 2002; 15: 5-26.

18.- Broide J, Soferman R, Kjvitv $S$, et al: Low-dose adrenocorticotropin test reveals impaired adrenal function to patients taking inhaled corticosteroids. J Clin Endocrinol Metab 1995; 80: 1243 -6. 
19.- Kelly HW, Nelson HS: Potential adverse effects of the inhaled corticosteroids. J Allergy Clin Immunol 2003; 112: 469-78.

20.- Patel E, Wales JK, IKibirige MS, Massarano AA, Courjel JM, Clayton PE: Symptomatic adrenal ir, sufficiencv during inhaled corticosteroid treatment. Arch Dis Child 2001; S5: 330-4.

21.- Drake AJ, Howells RJ, Shield JE, Prendiville A, Ward PS, Crowne FC: Symptomatic adrenal insufflciency presenting with hypoglycaemia in children vñth asthma receiving high dose inhaled fiuticasone propionate. UMJ 2002; 324: 1081-3.
22.- World Flealth Organization: Fluticasone and adrenal crisis. Aust Adverse Drug React Buil 2003; 22: 6.

23.- Crowley S, Hindmarsh PC, Holownia P, et al: The use of low doses of ACTH in the investigation of adrenal function in man. J Endocrinol 1991; 130: 475.

24.- Mellis CM, Phelan PD: Asthma deaths in children a continuing problem. Thorax 1977; 32: 29-34.

25.- Drake AJ, Howells RJ, Shield JPH, Prendiville A, Ward $P S$, Crowne EC: Symptomatic adrenal insufficiency presenting with hypoglycaemia in children with asthma receiving high dose inhaled fluticasone. BMJ 2002; 3245: 1081-2. 